\title{
Sibling screening in suspected abusive head trauma: a proposed guideline
}

\author{
Kshitij Mankad ${ }^{1} \cdot$ Jai Sidpra ${ }^{2} \cdot$ Adam J. Oates $^{3} \cdot$ Alistair Calder $^{1} \cdot$ Amaka C. Offiah $^{4} \cdot$ Arabinda Choudhary $^{5}$
}

Received: 22 July 2020 / Revised: 29 September 2020 / Accepted: 16 November 2020 / Published online: 12 May 2021

(C) Springer-Verlag GmbH Germany, part of Springer Nature 2021

\begin{abstract}
Abusive head trauma (AHT) is the leading cause of death from child abuse in children younger than 5 years. It is well documented that the infant contacts of children presenting with suspected AHT are at an increased risk of abuse when compared to the general infant population. Despite this association, a paucity of literature stratifies this risk and translates it to the clinic such that this high-risk group is stringently screened for abusive injuries. In this light, the authors propose a standardised screening method for all contact children of the index case and call for further consensus on the subject.
\end{abstract}

Keywords Abusive head trauma $\cdot$ Child abuse $\cdot$ Children $\cdot$ Infants $\cdot$ Screening $\cdot$ Siblings

\section{Introduction}

Abusive head trauma (AHT) as a term represents a constellation of craniospinal injuries precipitated by a forceful insult to the head, whether by shaking, impaction or a combination. It is a disturbingly common entity (prevalence 20-30 in 100,000 children) and the predominant cause of morbidity and mortality in children younger than 2 years with traumatic brain injury [1]. As such, it weighs a heavy personal and financial burden on society.

Siblings of abused children younger than 2 years, particularly those of multiples birth, are themselves at an increased risk of abuse in comparison to the general population. Despite this recognised association, a paucity of literature outlines the degree to which these children are at risk and how they should

Kshitij Mankad

kshitij.mankad@gosh.nhs.uk

1 Department of Radiology, Great Ormond Street Hospital, Great Ormond Street, London WC1N 3JH, UK

2 University College London Medical School, London, UK

3 Department of Radiology, Birmingham Children's Hospital, Birmingham, UK

4 Academic Unit of Child Health, University of Sheffield, Western Bank, Sheffield, UK

5 Department of Radiology, University of Arkansas for Medical Sciences, Little Rock, AR, USA be assessed by the physician [2]. Campbell et al. [3] reported a survey of 93 respondent child abuse physicians in the United States, of whom $40 \%$ reported routine conflict surrounding the assessment of contact children. This lack of unanimity, in addition to the poor outcomes associated with missed cases of AHT, further the need for the stringent evaluation of these children [4]. In this light, we propose a guideline for assessing the siblings of children with suspected AHT and call for further consensus on the subject.

\section{Siblings and risk: what is the evidence?}

Several observational studies note the increased prevalence of AHT and wider domestic physical abuse in the siblings of abused children. Hamilton-Giachritsis and Browne [5] studied 795 siblings of 400 index cases of abused children and identified that, in $37 \%$, abuse was not focused but directed towards all children whilst, in $20 \%$, abuse was specifically directed towards one or more children. Importantly, no paternal or infantile risk factors were characterised that distinguished perpetrators who abused all children from perpetrators who abused specific children [5]. A better understanding of these influencing factors and the motives of the perpetrator might permit further risk stratification and improve the identification of high-risk siblings.

Recognised risk factors for abuse related to the child include age, multiple-birth siblings and chronic disease. Lindberg et al. [6] conducted a study of 134 contacts of abused 
children who underwent skeletal survey and found at least 1 abusive fracture in 16 (11.9\%) of these contact cases. None of these fractures was evident on external clinical examination and so these data support the use of skeletal survey in all potentially abused children younger than 1 year independent of clinical findings [6]. Importantly, twins of abused children were more likely to be abused than non-twin siblings, with an odds ratio of 20.1 (95\% confidence interval 5.8-69.9) [6]. This notion of twins being at a relatively increased risk of abuse is furthered in the wider literature [7, 8]. Children with chronic disease are additionally predisposed to abuse and so particular care should be taken in screening these individuals $[9,10]$.

Parental vulnerabilities - notably mental health disorders, intellectual disability, substance abuse and financial difficulties - have also been documented as risk factors for abuse of both the index case and other children being cared for in the same environment $[11,12]$.

\section{Current and proposed imaging guidelines}

Despite the well-described increased risk of abusive injuries in contacts of abused children, the screening of these contacts is relatively infrequent and is not risk-appropriate. Indeed, a study of 1,918 contacts identified abusive injuries in $9.4 \%$ and found that one or more recommended imaging modalities were omitted in greater than $20 \%$ of cases [8].
The United Kingdom's Royal College of Radiology (RCR) offers the most comprehensive guidance for assessing contact cases, recommending that all multiple-birth siblings younger than 2 years have the same indicated imaging as the index case and that age-appropriate imaging be considered on a case-bycase basis for all siblings and children younger than 2 years who are cared for by the suspected perpetrator, whether in the same household or elsewhere [13]. The RCR also advises that, if these guidelines are not followed, senior clinicians document reasons for this in the patient's medical record. The Royal College of Paediatrics and Child Health (RCPCH) echoes this guidance and recommends imaging siblings younger than 2 years who have external evidence of physical abuse and to consider imaging siblings if there is any suspicion of abuse for other reasons [14].

Although otherwise comprehensive, the American College of Radiology's appropriateness criteria for suspected physical abuse of the child lack consideration of the siblings of children presenting with suspected AHT [15]. Other guidelines and statements similarly lack recommendations for children other than the index case [16-18]. Our proposed assessment guideline is presented in Fig. 1.

In addition to a comprehensive clinical examination of all siblings as per the child protection protocol, we propose a skeletal survey for all contacts younger than 2 years. Children older than 2 years with evidence or suspicion of trauma should undergo targeted skeletal imaging as indicated by their clinical findings. Skeletal survey is not indicated in this age group because of its relatively low-yield detection of

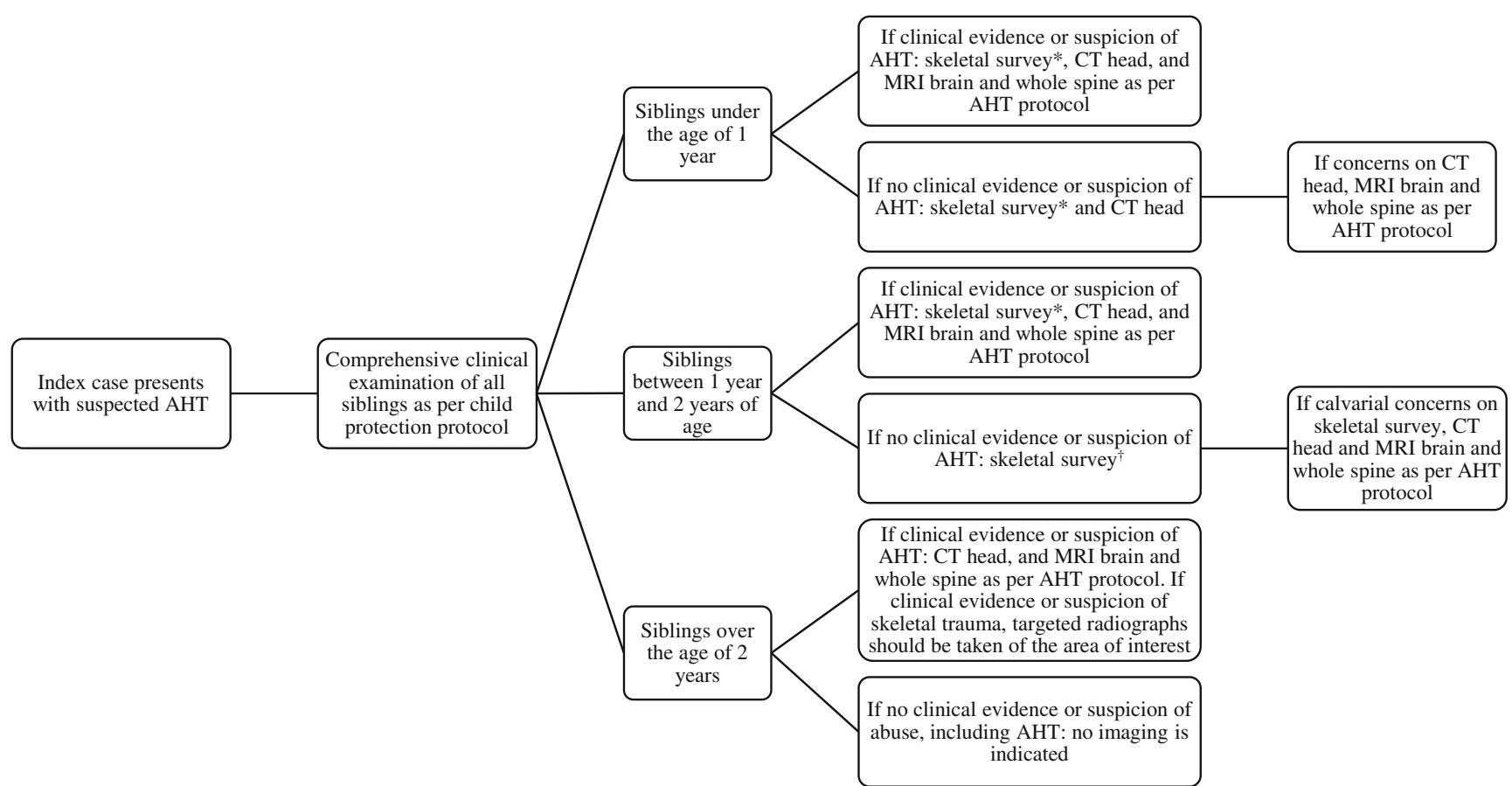

Fig. 1 Proposed guideline for assessing contacts of children with suspected abusive head trauma (AHT). *Skeletal survey should omit skull radiographs in favour of head CT. 'Skeletal survey should include skull radiographs 
undiagnosed fractures in light of the considerable dose of additional radiation $[19,20]$. We, however, note that full skeletal survey might be of benefit in select children older than 2 years, for example children with communication difficulties who are unable to give an accurate history. The potency of skeletal surveys for detecting abusive fractures is well proven in the literature. A retrospective study of 2,036 children younger than 60 months with skeletal surveys performed to evaluate for suspected abuse identified fractures in $18 \%$ of children [20]. A similar study of 703 skeletal surveys of suspected child abuse victims identified fractures in $10.8 \%$ of children, with those younger than 6 months having the highest rates of positive skeletal surveys [21]. These data corroborate the use of skeletal survey in the detection of acute and healing fractures in children. If the child is to have a CT head, then skull radiographs can be omitted from the skeletal survey, though this is an emerging view and future studies on this area are warranted [22]. Follow-up skeletal survey is a similarly powerful though not invariably used tool. In a study of 1,470 children, Singh et al. [23] found that only 169 underwent a follow-up skeletal survey but that, when performed, previously unrecognised abusive fractures were detected in $14 \%$ of cases.

We additionally recommend a computed tomography (CT) scan and magnetic resonance imaging (MRI) of the brain and whole spine, as per the AHT protocol, in all contacts where there is evidence or suspicion of trauma including neurologic symptomatology. Contrastingly, even in the absence of neurologic signs or symptoms, children younger than 1 year in whom abuse is suspected should have a CT scan of the head and a subsequent MRI if the CT raises concerns. The use of neuraxial MRI in children with likely abuse is advised because it is the most sensitive modality for detecting extra-axial (subdural) collections, parenchymal injuries, cerebral oedema, and the evolving neuroimaging features of acute or preceding AHT [24]. CT scans of the head are not as specific but they are a faster and less resource-intensive modality that permits unrivalled assessment of the calvarium for potential skull fractures $[25,26]$. For this reason, CTs are best suited to a screening role.

Children older than 2 years with no evidence or suspicion of trauma require no skeletal survey or neuroimaging.

\section{Ongoing challenges}

The challenges of imaging siblings vary across institutions depending on how they manage the index child with suspected abuse. This is a particular challenge in the setting of a patient presenting after routine working hours, when the resources of child abuse paediatricians, sedation providers or radiologic expertise might not be readily available. Points of contention include:
1. In the scenario of a patient presenting after routine working hours who is admitted for further workup (including advanced imaging on the next working day), what should be the management and workup of appropriate-age siblings?

2. If the skeletal survey or CT head is performed to exclude abuse at presentation in the emergency department, but these are negative and the child is sent home, then when and what should be the workup of the patient's siblings?

3. Given the expense of additional imaging, the cost and risk of sedation if required, and the diagnostic yield of imaging the siblings, physicians are able to recruit resources for the index patient but, for asymptomatic siblings, asking for additional resources can prove difficult.

Preliminary and anecdotal data suggest there has been an increase in the incidence of AHT during the prolonged lockdown period enforced in many nations during the severe respiratory syndrome coronavirus 2 (SARS-CoV-2) pandemic (coronavirus disease 2019 [COVID-19]) [27]. This phenomenon is historically reflected in other tumultuous periods with heavy associated psychosocial and socioeconomic burdens, for example following severe natural disasters [28]. Whether this is substantiated in more rigorous analyses remains to be seen, but these data highlight the importance of having a protocol for assessing siblings because clinical evaluation can be more problematic if there is a delay in the presentation of children with suspected AHT.

\section{Conclusion}

The involvement of a multidisciplinary team and clear communication with child protective services are imperative. In this schema, the radiologist plays a central role in identifying the hallmarks of potential abuse and in conveying this opinion, as well as the degree of certainty with which this position is held.

The assessment of contact children should be performed with the same care and rigour as that given to the index case. It is our hope that this proposed guideline aids in the evaluation of these children. A consensus-driven best practice approach for siblings of an abused infant is expected to help child abuse paediatricians and radiologists deliver optimum care and justify the demand for extra resources. We have established the framework and begun the dialogue upon which this consensus can be built.

\section{Compliance with ethical standards}

Conflicts of interest None 


\section{References}

1. Parks S, Sugerman D, Xu L et al (2012) Characteristics of non-fatal abusive head trauma among children in the USA, 2003-2008: application of the CDC operational case definition to national hospital inpatient data HHS public access. Inj Prev 18:392-398

2. Campbell KA, Squires J, Cook LJ et al (2009) Disparities in the medical examination of children in the home of a child with suspected physical abuse. Child Abuse Negl 33:612-617

3. Campbell KA, Bogen DL, Berger RP (2006) The other children: a survey of child abuse physicians on the medical evaluation of children living with a physically abused child. JAMA Pediatr 160: 1241-1246

4. Jenny C, Hymel KP, Ritzen A et al (1999) Analysis of missed cases of abusive head trauma. JAMA 282:621-627

5. Hamilton-Giachritsis CE, Browne KD (2005) A retrospective study of risk to siblings in abusing families. J Fam Psychol 19:619-624

6. Lindberg DM, Shapiro RA, Laskey AL et al (2012) Prevalence of abusive injuries in siblings and household contacts of physically abused children. Pediatrics 130:193-201

7. Becker JC, Liersch R, Tautz C et al (1998) Shaken baby syndrome: report on four pairs of twins. Child Abuse Negl 22:931-937

8. Lindberg DM, Blood EA, Campbell KA et al (2013) Predictors of screening and injury in contacts of physically abused children. $\mathrm{J}$ Pediatr 163:730-735

9. Krug EG, Dahlberg LL, Mercy JA et al (2002) World report on violence and health. World Health Organization. https://www.who. int/violence_injury_prevention/violence/world_report/en/. Accessed 2 Sept 2020

10. Paddock M, Sprigg A, Offiah AC (2017) Imaging and reporting considerations for suspected physical abuse (non-accidental injury) in infants and young children. Part 1: initial considerations and appendicular skeleton. Clin Radiol 72:179-188

11. Schnitzer PG, Ewigman BG (2005) Child deaths resulting from inflicted injuries: household risk factors and perpetrator characteristics. Pediatrics 116:e687-e693

12. UK Department for Education (2019) Characteristics of children in need: 2018 to 2019. Gov.uk website. https://www.gov.uk/ government/statistics/characteristics-of-children-in-need-2018-to2019. Accessed 2 Sept 2020

13. Society and College of Radiographers and the Royal College of Radiologists (2018) The radiological investigation of suspected physical abuse in children. RCR online document. https://www. rcr.ac.uk/system/files/publication/field_publication_files/bfcr174_ suspected_physical_abuse.pdf. Accessed 2 Sept $20 \overline{20}$

14. Royal College of Paediatrics and Child Health (2019) Recognition of physical abuse. In: Child protection companion. RCPCH website. https://childprotection.rcpch.ac.uk/child-protectioncompanion/. Accessed 2 Sept 2020

15. Wootton-Gorges SL, Soares BP, Alazraki AL et al (2017) ACR appropriateness criteria: suspected physical abuse - child. J Am Coll Radiol 14:338-349

16. Government of Western Australia (2014) Diagnostic imaging pathways - paediatric, injury (non-accidental). Diagnostic Imaging Pathways online document. http://www.imagingpathways.health. wa.gov.au/index.php/imaging-pathways/paediatrics/nonaccidental-injury\#pathway. Accessed 2 Sept 2020

17. Choudhary AK, Servaes S, Slovis TL et al (2018) Consensus statement on abusive head trauma in infants and young children. Pediatr Radiol 48:1048-1065

18. Narang SK, Fingarson A, Lukefahr J et al (2020) Abusive head trauma in infants and children. Pediatrics 145:e20200203

19. Paine CW, Wood JN (2018) Skeletal surveys in young, injured children: a systematic review. Child Abuse Negl 76:237-249

20. Lindberg DM, Berger RP, Reynolds MS et al (2014) Yield of skeletal survey by age in children referred to abuse specialists. J Pediatr $164: 1268-1273$

21. Duffy SO, Squires J, Fromkin JB et al (2011) Use of skeletal surveys to evaluate for physical abuse: analysis of 703 consecutive skeletal surveys. Pediatrics 127:e47-e52

22. Martin A, Paddock M, Johns CS et al (2020) Avoiding skull radiographs in infants with suspected inflicted injury who also undergo head CT: "a no-brainer?" Eur Radiol 30:1480-1487

23. Singh R, Squires J, Fromkin JB et al (2012) Assessing the use of follow-up skeletal surveys in children with suspected physical abuse. J Trauma Acute Care Surg 73:972-976

24. Kemp AM, Rajaram S, Mann M et al (2009) What neuroimaging should be performed in children in whom inflicted brain injury (iBI) is suspected? A systematic review. Clin Radiol 64:473-483

25. Choudhary AK, Jha B, Boal DK et al (2010) Occipital sutures and its variations: the value of $3 \mathrm{D}-\mathrm{CT}$ and how to differentiate it from fractures using 3D-CT? Surg Radiol Anat 32:807-816

26. Culotta PA, Crowe JE, Tran Q-A et al (2017) Performance of computed tomography of the head to evaluate for skull fractures in infants with suspected non-accidental trauma. Pediatr Radiol 47: $74-81$

27. Sidpra J, Abomeli D, Hameed B et al (2020) Rise in the incidence of abusive head trauma during the COVID-19 pandemic. Arch Dis Child. https://doi.org/10.1136/archdischild-2020-319872

28. Campbell AM (2020) An increasing risk of family violence during the Covid-19 pandemic: strengthening community collaborations to save lives. Forensic Sci Int Rep 2:100089

Publisher's note Springer Nature remains neutral with regard to jurisdictional claims in published maps and institutional affiliations. 